\title{
König Sigismund August von Polen und seine evangelischen Hofprediger.
}

\author{
Von Lic. Dr. Theodor Wotsohke.
}

Seit den dreiBiger Jahren war die reformationsfreundliche Haltung des jungen polnischen Königs Sigismand August allbekannt. ${ }^{1}$ ) Die Freunde des Erangeliums erhofften, die Altgläabigen befurchteten seinen baldigen offenen AnschlnB an die erneuerte Kirche. Seit dem Spätsommer 1543 residierte er mit seiner jugendlichen Gattin Elisabeth von Österreich in Wilna, während sein Vater, König Sigismund, in Krakan Hof hielt. Die räumliche Trennung von dem reformatorischen Einflussen wobl nicht ganz verschlossenen, aber doch römisch gesinnten Kreise, der seinen Vater amgab, und die Unabhängigkeit, die er als selbständiger Grobfurst von Litauen 1544 erhielt, ermöglichten ihm, deutlich seine Hinneigung za den Wittenbergern za bekunden. Fr sprach offen von der Notwendigkeit einer Reformation and förderte die Anhänger Lathers. ${ }^{2}$ ) Seine Hofprädikanten ließ er in durchaus evangelischem Sinne predigen; sie griffen auf die heilige Schrift zuruck und geißelten die Schäden der Hierarchie, wie sie schlimmer noch als anderwärts in Polen und Litanen zatage traten. Im Vertranen anf seine antirömische Haltung konnte Herzog Albrecht von Preußen Fruh-

1) Vgl. Cochläus an Vergerio unter dem 27. Juli 1537: „Etiam non semel audivi iuniorem regem, hoc est filium regis Poloniae, lutherico fermento infectum esse ${ }^{\text {. }}$. W. Friedensburg in der Zeitschrift für Kirchengeschichte Bd. 18 S. 254.

3) So konnte Herzog Albrecht z. B. dem jungen Könige den ob seines erangelischen Glaubens rerfolgten Litauer Georg Zablocki empfehlen. Vgl. Wotschke, Abraham Culvensis. Altpr. MLonatsschrift Bd. 42 S. 171.

Arcbir for Reformationsgeschlchte IF. 4. 
jabr $15+6$ ihn für die schmalkaldischen Verbündeten za gewinnen suchen. Die Einzelheiten der Verhandlungen, zu denen der Herzog im Mai nach Wilna geeilt war and die persönlich gefuhrt wurden, entziehen sich unserer Kenntnis. Wir wissen nur, dab der junge König den Protestanten wohlwollende Neutralität zusicherte, anch auf seinen Vater einzawirken rersprach, daB Polen dem Kaiser keine linterstutzang leiste. ${ }^{1}$ ) Wir wissen ferner, daB der Jagellone gern dem Glaubenszeugnis des bekenntnisfreudigen Hohenzollern sein Ohr geliehen hat. Herzog Albrecbt rersprach anter anderem, alsbald nach seiner Ruckkehr nach Königsberg ihm einige evangelische Schriften zar weiteren Einfuhrung in die Erkenntnis der biblischen Wahrheit za senden. Am 19. Jali schickte er für ihn an den Hofmeister der Königin Gabriel Therla (Tarlo) ein Gebetbuch, das Augsburger Bekenntnis and Melanchthons loci dentsch und lateinisch. ${ }^{2}$ ) Auch in den folgenden Jahren versorgte er ihn fleiBig mit evangelischen Schriften.

Noch deutlicher als aus diesen gern hingenommenen Gaben ersehen wir des jungen polnischen Königs Hinneigung zar Reformation aus der Lnterstutzang und Förderang, die

1) Wilna, den 31. Jai 1546 schrieb der Herzog im Einverständnis mit Sigismund August an den alten König, nachdem er des Konzils zu Trient gedacht, unter anderem: „So bitt ich auff beiligendt credentz wegen aller churfiirsten, grafenn, herren, stedt rad stend, so der christlichen religion rerwandt, gantz diust- rnd ireuntlichenn, E. K. I. wolle die rmbstende disser hendel beuorab, wes sachen dis seindt rnd wes ehre (als des lieben gotts) es angehet, daneben wol erwegenn, das die euangelischen mit götlicher schrift jres christlichen vorhabens bericht rnnd gewiesenn jnnewerden, bitten rnd begeren, dem auch, worin sye wie gemelt rberwisenn, zuweichen vnd stadt zugeben mebr dann einmabl vberbittigk gewesenn vnd noch. Ierhalben sich, ob gleich darumb auf gemachte parteyische beschlıb. durch die geistlichkeit oder andere etwas an E. K. M. denn euangelischenn $\mathbf{z w}$ beschwer gelangete, in nichts bewegen oder anfbringen lassen, dergleichen auch nit gestattenn, das E. K. M. vnderthan wider die euangelischen dienen, sunder vilmehr als ein liebhaber des fridens die mittel vnd wege, so zu gots ehre, fried, ruhe vnd einigkeit dinstlich. suchen, ferdern vod vortstellen, damit der liebe friedenn jn der christenheit erhalten ${ }^{4}$. Vgl. ferner den Brief des Hosins an Dantiskus Krakau, den 19. Juni 1546. Hosii epistolae I S. 227.

2) Vgl. Beilage 1. 
er Herzog Albrecht lieh, als dieser dem alten Könige den Trost des erangelischen Glaubens bringen wollte. Hochbetagt ging Sigismund I. dem Grabe entgegen. Lm des Gewissens willen meinte der Lehnsfurst rerpflichtet zu sein, den greisen, hinfälligen Herrscher hinzuweisen auf die Gnade Gottes, die ohn unser Verdienst und unsere Wurdigkeit un Christi willen gerecht macht, um also in ihm Frendigkeit zu erwecken auch fur die letzte schwere Stande. Am 20. Norember $15 \pm 6$ schrieb er an den König nach Krakau. Lm indessen nicht ohne Wissen and Willen des jungen Herrschers den greisen König religiös za beeinflussen, ließ er den Brief durch den genannten Gabriel Therla erst Sigismand Angast in Wilna rorlegen. ${ }^{1}$ ) Sofort erklärte sich dieser mit dem herzoglichen Schreiben einverstanden. Wenn darauf Polens Großkanzler Thomas Sobocki seinem königlichen Herrn in Krakau das Glaubenszengnis ans Königsberg vorlas, es mit ihm besprach, selbst die Rechtfertigung allein durch den Glauben vor ihm rertrat, so tat er es nicht nur ans eigener, 1525 in Wittenberg empfangener Glanbensuberzeugung, ${ }^{2}$ ) sondern auch aus Ergebenheit gegen den jungen Herrseher, der den Brief des Herzogs vom 20. November an ihn onterstutzt hatte. ${ }^{3}$ )

Der erste Hofprediger, der in Wilna ror Sigismund August von der Kanzel herab im reformatorischen Sinne wirkte, war Martin Gallinius. Leider wissen wir von ihm wenig mehr als den Namen. Er hat später auch mit seiner evangelischen Vergangenheit gebrochen, ist zurltckgekehrt zur römischen Kirche, von der er sich offen allerdings nie losgesagt hatte, und hat selbst in Krakan das Wort ergriffen gegen die Reformation and seine evangelischen Nachfolger im Hofpredigeramte. ${ }^{4}$ ) Aber noch in Italien, in Padua,

1) Vgl. Beilage 2, 3 und 4.

2) Thomas Sobocki ist am 10. Juni 1525 in Wittenberg immatriknliert worden. Melanchthonischer zucht vnd auch genut ${ }^{\mu}$ charakterisiert ihn der bekanute Krakauer Ratmann und Schriftsteller Jost Ludwig Dietz. Vgl. Wotschke, Abraham Culrensis S. 177.

3) Vgl. Beilage 5.

4) Vgl. das Schreiben des Augustinos Rotundus an Hosius rom 7. Jai 1549: „Yartinus Gallinius, qui nuper est ex Italia reversus et ipse egregiam in coarguendis istis neochristianorum erroribus operam 
wo wir ihn 1547 sehen, hat er aus seiner protestantischen Gesinnung kein Hebl gemacht. ${ }^{1}$ )

Etwa 1547 hatte Sigismund August zu seinen Hof. prädikanten Johann Cosmius aud Lanrentius Diskordia berufen. ${ }^{2}$ ) Nach einer allerdings nicht einwandsfreien Nachricht soll sie der Bischof Lchanski. der bekanntlich anlänglich reformatorisch gesinnt war, empfohlen haben. ${ }^{3}$ ) $\ddot{\text { ïber }}$ die Vorgeschichte beider Prädikanten sind wir nur wenig unterrichtet. Nach Wengierski soll Cosmins frther Erzieber der Söhne des Posener Hauptmanns Grafen Andreas Gorka gewesen sein. Laurentins hat 1539 an der Jagellonischen Universität zu Krakau studiert. ${ }^{4}$ Mitte der vierziger Jabre begegnet er uns als Prädikant an der Aula des Litauers Stanislaus Kieyzgalo, der ihn Herbst $15+6$ nach Königsberg

navat, concionatus ter in arce est, toties in parochiali Divae Virginis. Sed istorum onınia hic sordent, quae rel Cosmii rel Discordiae calculo non probantur". ¿̈brigens hat Rotundus, der spätere Vogt von WVilna, der Freund des Hosius und eifrige Gegner der Refornation. in Wittenbers studiert. Im April 1539 hat er sich an der cathedra Lutheri immatrikulieren lassen.

1) Johann Maczinski, der Schüler Melanchthons, der ron Wittenberg nach Zürich nud von hier nach Italien gezogen war, schreibt Padua, den 3. MIärz 1547 an Konrad Pellikan: „De rege inniore communis persuasio est hominum ipsum prorsus esse lntheranum. Habemus hic nobiscum Patavii concionatorem illins, rirum certe doctum in sacris literis et synceriori religione exercitatum olim etiam monachun et per unultos annos in monastico babitu docentem, nunc nihil commune habet cum monachis, cucullum rege sic rolente et pro eo apud pontificem Romanum intercedente reiecit, non gaudet longo et sinuosu restitu papistico, barbaram promissam alit, quod ceteris papistis apul nos religio est. Venit anten in Italiam propter calculum, a quo iam est liberatus opera chirurgi Patavini. Parat igitur reditum in Poloniam. nam et rex illi scribit, ut eo citius redeat et docendi evangelii munus, jnquit rex in literis, reassumat".

2) Nach den von Dalton veröffentlichten Synodalprotokollen hätten sie freilich schon seit 1544 in Wilna gewirkt.

3) In der Instrultion, die Mai 1051 den Gesandten des Krakauer Kapitels gegeben worde, heißt es ron Uchanski: ${ }_{r}$ Cosmium et Discordiam in Lituaniam miserat, qui curiam regis et totam Lituaniam iufecerant ${ }^{4}$. Acta hist. res gestas Poloniae illustrantia I. Cracoviae 1878 S. 477.

4) Als Laurentius Johannis de Prasnys dioc. Plocensis begegnet er uns im Universitätsalbum. 
an Herzog Albrecht abgeordert hatte ${ }^{1}$ ) und wenig später den jungen Könige empfohlen haben mub. Beide Prädikanten vertraten mit Entschiedenheit den reformatorischen Standpunkt, lehrten die Rechtfertigung allein durch den Glanben und eiferten gegen die römischen Mißbräuche. Mit reichen Gaben ausgestattet und tuchtige Redner, fesselten sie außer dem königlichen Hofe die Bürgerschaft Wilnas, ja den Adel ron ganz Litanen an ihre Kanzel. Ihnen haben wir es als Verdienst zuznerkennen, da $B$ auch nach dem Tode Rapagelans und Culrensis 1545 der warme Fruhlingswind der Reformation durch die erstarrte Kirche im fernen Osten wehte.

Mit Schrecken nahmen es die Bischöfe wahr, wie nnter dem Einflusse der Hofprediger der junge König und seine l'mgebang immer offener and entschiedener sich der Reformation zaneigten, and der Grobkanzler des Reichs, der Nachfolger des fur Polen and die Reformation leider nar allzafrth verstorbenen Thomas Sobocki, Samuel Maciejowski, zugleich Bischof von Krakan, griff zar Feder und richtete an Sigismand Angust ein Bitt- and Mahnschreiben, in dem er ibn beschwor, die ketzerisehen Prädikanten zu entlassen and der römischen Kirche treu zu bleiben. Wilna, den 24. Mai 1547 antwortete der Herrscher. Er verteidigt seine Prediger gegen den Vorwurf neuer Lehren, nur gegen die Mißbräuche and herrschende Sittenlosigkeit eiferten sie. ${ }^{2}$ ) Dann weist der Konig anf die religiose und geistliche Verwahrlosung hin, in der die Kirche die Bevölkerung gelassen habe. Noch herrsche in Litanen versteckt und offen das Heidentam. Das niedere Volk zumal sähe in Eichen, Linden, Bächen, Steinen and Schlangen Gottheiten, bete sie an and bringe ihnen Opfer; es sei daram ein Segen und wahrhaft christliches Bemthen, wenn seine Prädikanten den Glauben predigten and von dem toten Opferdienst zu Jesu Christo hinfuhrten. ${ }^{\text {? }}$ Maciejowski gab sich mit diesem Bescheide

1) Vgl. Wotschke, Abraham Culvensis S. 194.

2) "Negari non potest, quin in mores, abusus et vitia ntriusque ordinis plerumque inveherentur."

$\left.{ }^{3}\right)$ nExtra nrbem Vilnensem potissimum vero in Samogitia, ut ceteras saperstitiones taceamus, plebs rudis et inculta silvas, quercus, tilias, lapides denique serpentes pro diis colit eisque publice et privatim 
nicht zufrieden. Einige Monate später sandte er den bekannten Martin Cromer nach Wilna, ${ }^{1}$ ) doch rermochte auch dieser geschickte Diplomat nichts auszurichtell. ,.Jammernswert ist der Zustand des königlichen Hofes! Weun Gott sich unser nicht erbarmt, ist es um uns geschehen," schreibt am 6. Februar des folgenden Jahres Hosius aus Petrikau seinem Freunde und Koadjutor Johann Dantiskus. ${ }^{2}$ )

Als am ersten Osterfeiertage 1548 der alte König seine Augen schloB, erwarteten die Erangelischen in Polen allgemein, daB Sigismund August jetzt offen seinen Anschluß an die Reformation vollzieben und der Schutzherr der reinen Lehre im Osten Europas werden wuirde. Einen lebhaften Ausdruck lieh dieser Hoffnung der junge Hurnanist Jakob Kuchler in Posen in einer Zuschrift an den König. ${ }^{3}$ ) Aber die Erwartangen trogen. Politische Erwägungen, der Einflub, die Macht der Bischöfe, auch seine Abneigang vor einem festen entschlossenen Auftreten, hielten Sigismand August, „den König des morgenden Tages", ron einem Bruch mit der alten Kirche zartick. Bezeichnend ist es, dab er seine Hofprediger in Wilna zurucklieB, ${ }^{4}$ ) als er bei der Nachricht von dem Tode seines Vaters nach Krakau aufbrach. Einige Yonate später rief er sie freilich an seinen Hof nach Kleinpolen, nahm sie auch mit nach Petrikau auf den Reichstag, und Cosmins, der in dieser Zeit in einem Schreiben an den

victimas et holocausta offert ${ }^{\mu}$ etc. Das Schreiben des Königs liegt gedruckt vor. Hosii epistolae I S. 429.

1) Petrikan, den 21. August 1547 schreibt Hosius an Cromer: "Cansa, cur roceris, haec est, quod Vilnam te ad Regiam M Item mittere Rmus cogitat, ut istos, qui sibi soli sapere ridentur, quales quales verbi dei praecones in officio contineas.

2) Hosii epistolae I Nr. 279.

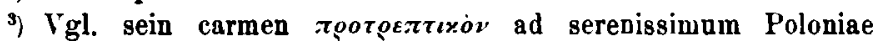
regem Sigismnndum Augustum pro purioris doctrinae evangelicae assertione. Wotschke, Jakob Kuchler, Zeitschrift d. hist. Gesellsch. Posen 1905 S. 224.

4) Stanislaus Bojanowski schreibt unter dem 12. Mai $15 \pm 8$ an Herzog Albrecht: „De religione quid sit sperandum nescio, sunt bona et mala signa. Episcopum Cracoviensem metuo, nos facimus, quae possumus. Regia Maiestas suos concionatores Vilnae reliquit, ut per vires in arce concionentur dem frauenzimmer (d. i. der Königin Barbara). Difficile est autem in hac re aliquid certi expiscari." 
Königsberger Professor Staphylus ron neuem seine unwandelbare Treue gegenüber dem Eravgelium betenert batte, ${ }^{1}$ ) durfte anfänglich auch wie ehedem predigen. ${ }^{2}$ ) Aber die Erbitterung, welche des Königs Heirat mit Barbara Radziwill nach dem Tode seiner ersten jugendlichen Gemahlin Elisabeth von Österreich in dem Kreise des Hochadels ge-

1) Vgl. das Schreiben des Staphylus an Herzog Albrecht bei Tschackert, Irkundenbuch zar Reformationsgeschichte des Herzogtums Preußen II Nr. 2163.

2) Petrikau, den 29. Dezember 1548 schreibt der herzogliche Agent Bernt Pohibel nach Königsberg: „Sonst weis ich E. F. G. besonders vit za schreiben, allein das junge kon• Majt herrn Kossumye, den die bischoffe allhier $\nabla f f$ die canzel nit haben wellen, das wort zu predigen gestatten. Bis nu am tage Steffani (26. Dez.) hat er anB koniglichem boffelich in jrer $\mathrm{Maj}^{\mathrm{t}}$ pallast in beywesen der herrn rethe rnd vier bischoffe als Gnysen, Posen, Croca vad Plotzke vnd ganzem hofgesint offentlich gepredigt rnd alle stende neben diesen geferlichen leufften mit vad durch gots wort trefflich vnderrichtet, welchs vielen trefflich zu hertzen hat gangen".

Anders lautet dagegen der Bericht des herzoglichen Gesandten Brandt rom 12. Dezember 1548 ans Petrikan. Nachdem er von den Folgen der Verehelichung des Königs mit Barbara Radziwill gesprochen, fährt er fort: „Menniglichen weis alhie, wie kon` Mlajt dem wortt zugethan gewesen vor diser verehlichnng, sunderlich aber so er des bischoffs von Cracan rhat vnd hülffe nicht bedorfft, ehe der bruder (Stanislaus Maciejowski, Kastellan von Lublin) hoffmeister wurde, aber itzund bett sich das wetter gantz vud gar vmbgewendett. also das es der bischoff so superstiose nicht fürgeben kan, that es der konig, dorüber sich ein iderer verwundertt. Es muB anch des koniges predicant nicht mer predigen, ist ein feiner gelerter man, tzwie bey mir zu gaste gewesen, habe in von der sachen dermassen reden horen vad wicht anders, dan wie man za Wittenbergk oder bey vns lerett. Er begeret von hinne, forcht, man werde inn vorgeben, der konig will im aber nicht erlauben rnd, wie ander leat dauon sagen, alleine darumb, das er allen beschaidt wais, wie es mit dieser verehlichnng zugangen. Letzlich hat er wollen baimlich sich von dannen machen, mich vmb rhat gefraget, habe ich im gerhaten, er solle es nicht thun, sunder

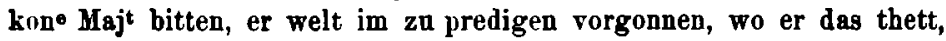
so konte er gote in seinem vaterlande wegen der zungen mer dineu dan bei E. F. D. Do es im aber nicht vergant wïrde, umb genedigen vrlaub anhalten, nicht dermassen als begerett er sich von konr Majt rud aus derselben dinst znwenden, sunder allaine an ein ortt sich zubegeben, do er schtudiren rnd sunst nicht mussig gehen dorffte, dohin zwinge in sein gewissen, so bald aber in kone Mlajt widderumb forderte, wolte er sich widder geschtellen. Dis het er sich gefallen lassen". 
weckt, die rerfehlte Politik des evangelischen Grafen Andreas Gorka, des ersten Magnaten des Reichs, sein unglaublich schroffes Auftreten gegen den Herrscher, trieb Sigismund August den Bischölen. im besonderen dem gewandten Samuel Maciejowski in die Arme. Schon um die Krönung seiner Gemahlin, ihre Anerkennung als seine rechtmäßige Gattin und Königin ron Polen za erhalten, darfte er jetzt mit der mächtigen Kirche sich nicht entzweien. Nan da der Adel fast geschlossen wider ihn stand, mußte er den Klerus sich rerpflichten. Bald erfahren die Hofprediger, daB zwar das Herz and die Glaubensuberzengung ihres Herrn sich nicht gewandelt habe, daß aber seine Politik andere Bahnen ging wie ehedem. Sie durften nicht mehr so oft und so frei predigen wie fruher, und wenn sie in alter Weise die Schäden der alten Kirche aufdeckten und eine Reformation forderten, so erhob sofort der jetzt bei dem Könige allmächtige Kanzler und Krakauer Bischof Samuel Maciejowski Einsprach. ${ }^{1}$ ) Zumal nach den Enththllungen des ehemaligen Hofpredigers Albinus ${ }^{2}$ ) wurden Cosmins und Laurentius Diskordia argwöhnisch beobachtet, beim Könige denunziert und dem Herrscher die Entziehung jeder Unterstutzang angedroht, falls er seinen Prädikanten nicht die Betätigung ihrer evangelischen Gesinnang verbiete.

In welch geistiger Unfreiheit Cosmius 1549 lebte, zeigt das Schreiben, welches er im Mai an Maciejowski richtete. Ein Priester, der offen zur Reformation ubergegangen and

1) Unter dem 12. Sfärz 1549 ersuchte ihn darnm das Krakauer Kapitel. Hosii epistolae I S. 307.

2) Am 1. Mai 1549 schreibt Bernt Pohibel dem Herzog Albrecht: "Uein her (Therla) hot wider mich vortrenlich gesagt, das sich die bischoffe neben andern kegen E. F. D. befaren, weil sie wissen, das konigliche Majt durch E. F. G. förderungen dem wort gots fast zagethan vad auch got lob gutten grundt vnd bescheidt wẹis, besorgen sich, es möchte jrem regimente schedlich seynn, so got alle sachen uach seinem gefallen ordnen vnd schicken würde. Auch wie ich von meynem hern vorstebe, so hot der losse mann, der Albinus, welcher zur Wilden königlicher prädicant gewesen, etzliche bïcher, so E. F. G. vnd andere irer Majestet zugeschickt, den pepstlichen angezeigt, dan sie $j m$ vortraudt worden $z$ vbersehen, aber nicht nachzaklaffen. In summa, der sathan schlefft nicht ${ }^{u}$. 
in die Ehe getreten war, wahrscheinlich der Pfarrer Valentin ron Krzezanow vier Meilen siddwestlich von Lablin, batte sich an ihn gewandt und ibn flehentlich gebeten, seinen Schritt mit Gründen der Schrift and Sätzen der Väter zu rechtfertigen, ihn ibberhaupt gegenuber der anhebenden Verfilgung unter seinen Schutz zu nehmen. Cosmius sagte ihm seine Unterstutzang $z \mathfrak{a}$, doch meinte der einst so einflubreiche Mann jetzt zuvor sich selbst den Rucken decken zu mulssen. Er schrieb an Maciejowski and setzte die Grunde auseinander, die ihn bestimmt hätten, des Angefeindeten sich anzunehmen, in feiner Weise dem Bischofe es nahelegend, nicht vorschnell zu urteilen, auf Grund des Wortes Gottes zu entscheiden und den Einflusterangen der Verleumder kein Gehör za schenken. ${ }^{1}$ ) In dem offenen Briefe, den er in derselben Zeit an alle Prädikanten und Priester richtete und den er unter dem 24. Mai 1549 eben diesem Maciejowski widmete, ${ }^{2}$ ) thergeht er ans demselben Grunde die falsche Lehre der alten Kirche, indem er die dogmatische Frage später in einer besonderen Schrift zu behandeln verspricht,") und legt nur den Finger anf das unsittliche, schändliche Treiben des polnischen Klerus. Eingehend deckt er die Schäden auf, an denen die Kirche unter dem lasterhaften Wandel der Priester leide, er zeigt, wie das bőse Beispiel des Klerus das Volk entsittliche, ${ }^{4}$ ) verrät ans, daB das

1) Deu Brief haben die Herausgeber der Briefe des Hosius im Anhange des ersten Bandes mitgeteilt. Vgl. Hosii epistolae I S. $445 \mathrm{f}$. Unter dem 24. MIai 1549 verwandte sich anch Stanislaus Orzechowski, der übrigens mit Cosmins in Brietwechsel stand, für den Prediger Valentin bei dem Krakaner Bischofe. Vgl. Korzeniowski, Orichoriana. S. 264 und 288.

2) Epistola Johannis Cosmii concionatoris S. R. M. ad ministros rerbi dei omneque adeo genus sacerdotnm, ut sese in ministerio praedicationis sinceros et in vitae officiis probos ac imitatione dignos praebeant. I. Cor. 9 Fratres, curn aliis proedicamus, ipsimet reprobi ne efficiamnr. Ibidem, Omnia faciamus propter evangeliam, ut participes eins efficiamur. Hinten: Datae Crac. ex suburbanis hortis d. Hechler, Calen. Junii anno domini 1549.

3) Vgl. S. B.: ${ }_{n}$ Xos de puritate doctrinae in verbo dei enarrando alio in loco de professo tractaturi sumus".

4) $\mathrm{S} . \mathrm{A}_{5}$ : ${ }_{n}$ Nos concionatores sacerdotesque, qui exemplum popnlo ad bene beateque vivendum esse debebamns, antores illi pessime ac 
schwelgerische and ehebrecherische Treiben der römischen Priester bei Griecben and Ruthenen sprichwörtlich geworden war. $\left.{ }^{1}\right)$ und fordert mit heiligem Ernste eine sittliche Erneuerung des Klerus, auch eine Lnterweisung des Volkes im Erangelium, eine schriftgemäße Predigt. Indem er jedoch hier von näheren Ausfuhrungen absieht. und von dem Hauptartikel des erangelischen Glaubens, der Rechtfertigang allein durch den Glauben. schweigt, auch bei aller Schärfe gegen den Klerus die Hierarchie nicht prinzipiell rerwirft, kann seine Schrift ebensogut als die Arbeit eines römischen Reformtheologen wie eines uberzeugten Protestanten gelten. Sie ist weniger ein Ausdruck des positiven Glaubensstandes unseres Polen, als ein Zeugnis des geistigen Druckes, unter dem er seit Anfang des Jahres 1549 seufzte und der ihm ein offenes Bekennen unmöglich machte bzw. nicht rätlich erscheinen lieb. ${ }^{2}$ )

Da das Buch wie anch der oben erwähnte Brief an Maciejowski nicht ron der königlichen Aula, sondern aus dem Garten Aichlers in der Vorstadt Krakaus datiert ist, scheint Cosmius Sommer 1549 seines Hofpredigeramtes

licentiose vivendi sunns. Morum enin nostrornm improbitas vitaeque etiam perperam ac praepostere actae iniqua institutio partim verbo dei seu adversa fronte repngnat, partim omnibus aliis hominibus in baratrum viciorum condemnationisque praeit. Sic fit, ut et verbo dei moribus nostris contumeliam facere rideamur et caeteris omnibus vitae nostrae pessimae contemplatione pessimo detrimento scandaloque sumu : $*$. s. $\mathrm{L}_{4}$ : "Liberi nostri heu illiberales spurio et non sacrosancto matrimonio suscepti adsunt nobis sacrificantibus, arrident nobis cantantibus. pulpita occupant nobis concionantibus, aurem nobis vellicaut de castitate disserentibus vel contra fornicationem clamantibus. ut hoc pacto et nos aliis ridendos propinemus et verbum dei in levitatis suspicionem ceu ludicrum quiddam adducere videamur".

1) $\mathrm{S} . \mathrm{H}_{4}$ : ${ }_{n}$ Certe Graeci ac Ratheni, quoties aliquem luxu et fornicatione difluere dicere rolunt, vivere eum ad instar sacerdotis latini dicunt ${ }^{\mu}$.

2) Auch das der Schrift beigegebene Gedicht eines Bekannten und Gesinnungsgenossen unseres Polen spricht nur im allgemeinen ron der Notwendigkeit einer Predigt des lauteren Evangeliums. Tgl. Sebasti Myonis carmen, in quo non offendantur boni et puri concionatores, sed saltem bi, qui pro verbo dei interdum vel historias vel sui capitis figmenta praedicant, rel genuinam sententiam rerbi dei violant. 
bereits enthoben gewesen za sein und seine Wobnung nicht mebr im königlichen schlosse gehabt zu haben. Jedenfalls trugen er und sein Amtsgenosse Laurentius sich mit dem Fedanken, Krakan zo rerlassen und anderwärts einen Wirkungskreis zu suchen. Aber die Hoffnung, daB die politische Lage sich ändern ond der König wieder seiner eigenen Überzeugung werde folgen können, dazn auch die Bitten vieler Freunde, za denen sich im Juli 1549 noch Laski gesellte, ${ }^{1}$ ) hielten sie schlieBlich wieder fest, vielleicht gaib ibnen anch der König die erbetene Entlassung nicht. Aber alle Hoffnungen auf einen Wandel der Verhältnisse trogen. Der König, der Laski auf seine verschiedenen Schreiben nicht einmal za antworten wagte, ${ }^{2}$ ) meinte immer mehr die Gunst und Unterstutznng der Bischöfe sich erkaufen zu milssen und nur im Bunde mit ihnen alle Schwierigkeiten der politischen Lage uberwinden zu können. Auf dem Reichstage zu Petrikan, der Mai 1550 anhob, forderte eine starke evangelische Partei freie Verkundigung des lanteren Gotteswortes and Aufhebung der geistlichen Jurisdiktion, aber anf sie sich zu stutzen nnd mit ibr die Kirche in Polen zu reformieren, wie er es einst in Wilna gedacht, scheint der König damals schon gar nicht mebr in den Bereich seiner Erwägnngen gezogen za haben.

In Petrikan erbat und erhielt Laurentius Diskordia seine Entlassung. Am 3. Juli 1550 ließ er sich von dem Grafen Gorka an Herzog Albrecht empfehlen. ${ }^{8}$ ) In Königsberg ubergab er dem Schutzherrn aller Evangelischen des Ostens, der ihn am 16. November an Panl Speratus nach Marienwerder sandte, einen heut leider verlorenen Brief und einige mir nicht näher bekannte Schriften seines Amtsgenossen Cosmius, unter ihnen vielleicht seine meines Wissens noch

1) Vgl. das Schreiben Laskis an Laurentius, welches Wengierski in seiner Reformationsgeschichte S. $212 \mathrm{f}$. mitteilt, irrtümlich aber in das Jahr 1552 verlegt.

2) Da der preußische Gesandte Brandt durch Krankheit an das Bett gefesselt war und für Laski nicht tätig sein konnte, bat Stanislaus Bojanowski den König um einen Bescbeid für ihn: ${ }_{n}$ Non est, quod responsum expectet," antwortete der König. „imo expectet, ego omnino volo respondere, sed vides, quod nunc commode non possum".

3) Vgl. Beilage 6. 
nicht wiederaufgefandene ,oratio ad Julinm III Pontificem Romanum pro indicendo libero et catholico concilio ac reformatione canonica in ecclesia facienda ", ${ }^{1}$ ) nach deren Erscheinen unserem Polen rom Könige ein strenges Schweigegebot auferlegt and der Drack unzensierter Schriften in Krakau unter hoher Strafe rerboten worden war. Am 11. Dezember 1550 sandte inm der Herzog seinen und seiner Gemahlin Dauk. ${ }^{2}$ ) Am Tage darauf tat in Krakan der König, der jetzt um der Krönnng willen jedem Winke des Gnesener Erzbischofes Nikolaus Dzierzgowski entsprach, den Schritt, der mit seiner reformationsfreundlichen Vergangenheit abschloB, ihn zum Werkzenge der Hierarchie werden und niederreißen lie $B$ was er einst selbst als Herrscher weiter auszubauen gedacht hatte. Er erlieb am 12. Dezember das bekannte, schon von Lubieniecki in seiner Reformationsgeschichte ${ }^{3}$ ) mitgeteilte Nandat gegen die Evangelischen in Polen, den Preis fur seine Krönung durch den Erzbischof. Wir müssen unserer Verwunderung Ausdruck geben, dab auch jetzt noch am königlichen Hofe einige Freunde der Reformation in ihrer Hoffnungsseligkeit verharren und meinen konnten, der König werde sich bald wieder frei machen von den Einflussen der Bischöfe und eine andere Politik einschlagen. "Weil ich got lob wol weis," schreibt am 9. April $15 \overline{0} 1$ Gabriel Therla aus Krakan, „das mein her durch gnad des almechtigen dem gothlichen wort wol zugethan, auch inn der gothlichen heiligen geschrifft genugsam erfahren, hab ich mich solcher

1) Nach Jochers polnischer Bibliographie ist die Schrift gedruckt .C Cracoviae apad viduam Floriani Unglerii $1550^{\mu}$ und ihr beigegeben -Henrici Schofferi epistola consolatoria ad d. Franciscum Stancarum captivum “. Empfohlen von der Königin Isabella von Ungarn, war Stancaro Ende 1549 nach Krakau gekommen und hatte durch Maciejowski die Professur der hebräischen Sprache an der Jagellonischeu Unirersität erhalten. Da er bei der Auslegung der Psalmen gegen das Anrufen der Heiligen eiferte, denunzierte ihn der Kanoniker und Gymnasiarch Nikolans Schadek bei dem Bischofe Maciejowski, der ihn darauf in Lipowitz einkerkern lieB. Vgl. Wotschke, Stancaros erster Aufenthalt in Posen. Histor. Monatsblätter für die Prov. Posen Bd. V S. $81 \mathrm{ff}$.

2) Vgl. Beilage 7 .

3) Lubieniecius, Historia reformationis Polonicae. Freistadii 1685 S. 60. 
mandaten nicht hart anfechten oder bekummern lassenn. Was gescheen, hat seyu masse. Ich weis gewis, das mir mein her mein gewissen rnd glauben nicht zwingen wirt. Das mag sich ein ander auch wol rorseheu. Die zeit mag auch rmlauffen, rnd welcher solch geschrifft ronn sich gebenn, hat auch macht ein andere zu geben."

Auch Cosmins scheint diesen Optimismus geteilt zu haben. Wir sehen ihn Anfang des Jahres $15 \overline{5} 1$ die Feder spitzen zu einer Schrift, die den Nacbweis fuhren sollte, $\mathrm{da} B$ in den reformatorischen Gemeinden die katholische und apostolische Kirche fortlebe, nicht aber in dem Papsttume, das mit seinen Mensehensatzungen und unbiblischen Ordnungen im völligen Gegensatze zu der alten christlichen Kirche stehe. Das königliche Mandat, das die katholische und apostolische Kirche schutzen wolle, sei deshalb gegen die Anhänger Roms zu rollstrecken, nicht gegen die Evangelischen, deren einziger Gedanke es sei, ihre Gemeinden nach dem Bilde der alten apostolisch-katholischen Kirche zu erneuern. Wegen der strengen Zensur in Krakau und der argwöhnischen Bewachung der Druckereien wollte Cosmius diese Schrift in Königsberg in Drack geben. Am 12. März schrieb er an den Herzog und bat um die Erlaubnis. ${ }^{1}$ ) Da Fruhjahr 1551 das viele Schneewasser die meisten Straben in Polen unwegsam gemacht hatte, scheint der Brief verspätet in die Hände des Fürten gekommen zu sein, and ehe er noch antworten konnte, erhielt er von seinem Agenten Pohibel ein Schreiben, das ihm von einem neuen Schlage der römischen Geistlichkeit wider unseren Polen berichtete: „Heute babenn dy pfaffen des kunigs prädicant her KoBomyn mit schwerer ladunge gefordert der meynunge, jnen alzo mit sonderen practiken rnnd lysten ron hynnen nach Lipowicz 3 meylen von Croca za senden. ${ }^{2}$ ) Die do hynn kommen, keren selten widderumb. Das ist dy große rrsach, das er diße zeit vor osternn sab atraque specie jm geheym vielen leuten gegebenn, dem hofgesinde Bo wol als anderenn, welche es begert habenn. Wywol er solchs eygner person nicht

1) Vgl. Beilage 8 .

2) In Lipowitz befand sich der bischöfliche Kerker. 
gethan, sonder hot eynen prister aus des hern Stagnitzky dorff nicht weyth ron Croca bey im gehalten, ${ }^{1}$ ) der solch ampt ausgericht. Erbeut sich eyner disputation, wie man wil sagen, ob es darzu komen, mag man jnne werden. E. F. (F. können abnbemen, was das gegebene maudat. ${ }^{2}$ ) $B_{0}$ E. F. G. eyn coper meynem heren zugeschickt. mit der zeit ror hipsche frachte bringen wird. Ist vor $d y$ geystlichen nicht gestanden, sonder sich an die Majt rnd ror jdermenniglich beruffen, wirt weyter was darans, bleibt E. F. G. rnverbaltenn. Ich merck, das ryll jm anhengig. Derhalbenn gebet es noch stille zu. Ire Majt nymbt sich nichts fast anu. ich halte, man were wol zufrieden, das es zur disputation keme, aber es ist jnen vngelegen. Got der schicks vordun zum besten, wirt was daraus, kombt wol an tag. Bitt E. F. (i. ganz vndertheniglich, da $B$ rnd anderes meyn schreiben dem feuer geben wellen, den was gescheen, hab ich aus meyns hern $^{3}$ ) munde, mir vff gudt vertrauen angezeigt . . E Eylent Croca, den 11 aprill gar am abent. Got weis, hab nit eher zur abfertigunge kommen megen."

Leider scheint Pohibel seinem Worte: „wirt weyter was daraus, bleibt E. F. G. vavorhalten « nicht entsprochen zu baben. Wir können seinen Berichten nichts Weiteres iiber das Schicksal des vor den Bischof zitierten Hofpredigers entnehmen. Ist Cosmius in dem Kerker zu Lipowitz lebendig begraben worden? Hat er anderwärts einen frühen Tod gefunden ${ }^{4}$ ) oder sich zurilckziehen können, wie es einst sein

1) Felix Cruciger, der Pfarrer in Niedzwiedz, dem Dorfe des Stanislaus Stadnicki, und spätere Superintendent der kleinpolnischen reformatorischen Kirche. Die Nachricht bei Dalton (Lasciana S. 398) post ferias paschatis fuit dispersio ministrornm propter metum canonicorum Cracoviensium. Felix Cruciger transtalerat se iu maiorem Poloniam ad d. Jarocz Ostrorog ${ }^{*}$ findet in Pohibels Schreiben ihre nähere Begründung.

2) Das königliche Mandat wider die Evangelischen rom 12. Dezember 1550.

3) Gabriel Therla, jetzt königlicher Vorschneider und Starost von Chelm, später Hofmeister der Königin Katharina von Österreich und Kastellan ron Radom.

4) Labieniecki S. 21 scheint anznnehmen, daß Cosmins in seinem Hofpredigeramte verstorben ist. 
Gedanke war, in einen stillen Wirkungskreis, ron dem wir keine Kunde haben?') Wir können diese Fragen nicht beantworten. Plötzlich wie sein Name in der polnischen Reformationsgeschichte auftaucht. rerschwindet er auch wieder.

\section{Beilagen.}

Die Urkunden sind sämtlich dem königlichen Staatsarchiv in Königsberg entnommen.

\section{Her\%og Albrecht an Gabriel Therla.}

Vnsern grus rnd gnedigen willen zuuorn. Edler rnd ernuester, besonder, lieber. Nachdem wir mit euch verlassenn, das wir der jungen könn Majt, vnserm gnedigen herren rnnd freuntlichen liebenn oheimenn, etzliche bucher zusenden wollen, demnach haben wir vir kleine buchlein zarichten lassenn, nemlich confessiones fidei rnd loci communes lateynisch rnd deutzsch, daneben anch ein bethbuchlein, in gnaden sinnend vnd begerend, ir wollet nach ewer bescheidenheit die buchlein irer kon Majt vleissiglichen veranthwurtten vnd irer konn Majt den bericht than, das die luci communes im lateinischen weither wie im teatschen, anmerkende, das solche vnnlängst im latein corrigiret, aber ins dentsche noch zar zeit nicht fertiret. So bald es aber wie rerhoflich ausgehenn wirt, sol kone Majt solchs vbersandt rnd zugefertiget werdenn. Inn dem bethbuchlein hoffenn wir, werde ire kone Majt ril gatter nutzer gebeth findenn. Weil dan der allerhöchst vmb erkentnus durchs gebeth gebethen sein will, so wollet zum anfahenn nach ewer bescheidenheit der konn Majt solchs erstlichenn vberantwartten, damit es ire kone Majt mit lust lesenn möge, den liebenn barmhertzigenn goth bittend, er wolle der konn Majt seinen heiligen geist rnd erkentnus seiner warheit gnediglichen verleyhenn ... Konigspergk, den 19. Juli 1546.

\section{Herzog Albrecht an Gabriel Therla.}

Jungsten eurem abscheid nach vberschicken wir junger konr Majt zu Polen, unserm gnedigen herrn rnd lieben oheimen, eine copienn, wes wir an alte kone Majt zu Polell geschriebenn, stellens jrer Majt, ob solche schriften vortgehen

1) Am 16. Dezember 1552 hat sich in Wittenberg ein Joannes Cosmo ex ducatu Lituaniae immatrikalieren lassen. Sollte dieser Student mit dem Hofprediger Johann Cosmius identisch sein? 
sollen oder nicht. anheimb. Nuhn sehen wir gantz gern, do die brif ann alten konigk mit rath junger konr Majt iren rortgang haben solten, das jr solche (als durch denn es am bequem rnd faglichsten beschehen konte) jnn gegenwertigkeit des groBcantzlers. ${ }^{1}$ ) welcher rnsers erachtens zu mehrerem bericht solchs handels nutz and dinstlich sein konnte, rberantwortten tettet. So es nu junger konr Majt dermassen rnd das es jm beisein des großcantzlers geschehe, dem wir dann darneben anch schreibenn vnd jnen mit treulicher ermanung vnd rathen anzuhaltenn gnediglich bittenn, rortzustellen gefielle, habt jr die brife an alte kone Majt zu euch zunehmen vnd zu eurer ankunft jn des obercantzlers gegen!wart, dem jr dann seinenn brif auch zuzastellenn, za rberantwarttenn, zu dem lieben got hoffende, darumb wir dann auch seine almechtigkeit mit vnserm sundhaftigen gebet $\mathrm{rn}$ aufhörlich anruffenn, er werde alle sachen zu ehrenn seines heiligen namens rnd worts zum bestenn schickenn. Stellen doch diesenn gantzenn handell, nichts weniger ob die rberreichung alter konr Majt brife im beyseinn des obercantzlers geschenn solle, jungen kon. Majt jnn derselben hohenn von got verlihenn vorstand vnd beschluB (wie dann dieselb dem jrer geschicklicheit nach wol zutun wird wissen) rnd jn euere bescheidenheit. Jm fahl, do ire kone Majt, das der großcantzler darbey sein solle, für vngerathen erwäge, so wollet vns denselben brif an grobcantzler lauttende widerumb vberschicken vnd das ander doch mit konr Majt rath jnn gottes nahmen vorstellenn. Do dan dasselbe auch fur vnratsam angesehen, vns solchs alles zuricksenden. Datam Konigspergk, den 20. Novembris 1546 .

\section{Herzog Albrecht an Thomas Sobocki.}

Wir seint verursacht worden, der alden konn Majt zu Polen, vnserm allerguedigsten hern, als dem der nuhmer alders halbenn of den grubenn gehet, allerlei zuschreiben, sonderlichen aber darumb, weil wir vus vodtertbenigen pflichten nach schuldigk erkennen, jrer könn Majt nicht allein inn weltlichenn, sonder anch inn geistlichenn sachenn rnser treuen nach des za rathen, des wir gegen got rnnd menniglichen zunerantwurtten, wie jr dan aus dem schreiben zuuernehmen. Seintemal wir danu eure personn anders nicht weder als einen christenn vermerkt, auch dergestalt kennen, das jr inn allen fellen koner Majt gern das ratet, das zur seelen heil dinstlicb, so sinnen wir an euch gantz gutlichen, jr wollet vnbeschweret sein, jrer konn Majt, das wir kurtz

1) Thomas Sobocki. 
gestellet. euer hohen bescheidenheit nach, als vil ronnöthen, erkleren rnd souil an euch der alden frommen seele, das sie mit Christo rnd seinem heiligenn engeln jnn ewigkeit ruben möge, rahten helffen. Daran erzeigt jr dem allerhochsten ein angenemes gattes werk, so seint wirs gnediglichenn ab\%anehmen gewogen. Dat. Konigspergk, den 20 . Norembris 1546.

\section{Herzog Albrecht an Sigismand Augast.}

Non dubium mihi est, quin S. R. Mtas V. in memoria recenti habeat, quae superiori tempore cum eadem de ratione statuque S. R. Mtis contulerim, quidque dictante et urgente conscientia mea de vera christiana religione, qua omnibus modis S. R. Mtem Suam imbutam cuperem, in medium attulerim. Ex adrerso antem, quae mihi tum temporis $\mathbf{S}$. $\mathbf{R}$. Mtas V. clementer iniungere utque in proposito perseverarem hortari dignata est, ea non modo memoria firmiter adhuc teneo, verum etiam pro fide et sabiectione mea debita diligentia exsequi sum conatus. Nam cum intelligam, mihi $s$. $\mathrm{R}$. Mtis V. consiliis et monitis acquiescendum eademque lege obstrictum est, ut quae ad S. R. Mtis senioris commodum, utilitatem, salutem corporis et animae, bona foelicitatemque pertinere cognoscam, diligenter ac fideliter pro viribus curare debeam, facere non potai, quin meam sedulitatem, debita servitia ac voluntatem bene merendi promptissimam in hac re ostenderem. Itaque hoc scriptum, cuius copiam hisce literis inserui, ad S. R. M. Suam destinavi. Quod ideo S. R. Mti V. mittendum videbatur, at ea et legeret prius et, quae sibi placeant, haec omnia pro summa sua prudentia et perspicatia indicaret. Quod si perlectis his atque probatis consultum S. K. Mti V. videbitur, at hoc modo illa etiam forma, quae mihi quidem optissima visa est et re vera christianae religionis meaeque fidei indubitata sententia est, S. R. Mti transmittatar, non inutile fore existimo, ut S. R. Mtas V. negocium nobili ac generoso sincere mihi dilecto d. Gabrieli Tharla, quocum nuper admodum hac ipsa de re locutus sum, quique hoc legationis hand gravate visus est suscipere, clementer iniungat ita tamen, ut illud ipsum scriptum S. R. Mti S. in praesentia magnifici d. supremi regni cancellarii, cui hoc etian nomine scribendum curavi, offert, non dubitans, utramque pro suo ingenio, prudentia et dexteritate id daturum operam, ut $S$. $R$. Mtas S. verum et nativum huius scripti mei sensum, qui ex ipsa rei veritate erutus est, percipiat. Quod ut fiat, S. R. Mti S. spiritus saneti donom et afflatum numinis ex corde intimo precor, et si quid porro eidem in hoc ipso religionis et animae salvandae negotio pro mea mediocritate 
servire aut commodare potero, ad id me et corpore et animo promptissimum offero. Regiomonte 20 . Norembris $15+6$.

\section{Thomas Sobocki an Herzog Albrecht.}

Scriptum Ill. Domnis Vrae ad S. R. YItem, quod in illis ad me literis inclusum fuit quodque Ill. Domtio Vra per me S. R. IIti legi roluit, eidem pro voluntate III. Domnis Vrae nemine tune alio praesente legi, quod et audirit diligenter et hoc Ill. Domnis Vrae officium, quo suum erga illam reram debitamque amorem testatur, animo grato suscepit. Ubi fait opus explicatione, eam non modo inter legendum, sed etiam per aliam occasionem adhibui. ita ut quae Ill. Domtio Vra scripsit, ea S. R. Mtas bene cognita et meditata habeat, quae et probat et in eis simul Illmae Domnis Vrae erga se debitum et propensum studium commendat. Nam qua est pietate, purum verbum dei et veram evangelii doctrinam labenter audit, instificationem suam ad solum Christam Jesum refert, in eo solo difisa omnibus viribus et meritis suis certam fiduciam et spem salutis aeternae ponit, in qua at magis ac magis confirmetur, saepe illa, quae ad hane rem pertinere seio, ei suggero ac commemoro. Dominus deus mentem et cor eius spiritu sancto illustret et confirmet, ut veram evangelii lucem plene cognitam et firma fiducia apprehensam ad extremum usque retincat ... Datum Cracoviae 1. Januarii 1547.

\section{Graf Andreas Gorka an Herzog Albrecht.}

Cum ita fatis nescio quibus comparatum sit, ut ecclesiam in hac extrema mundi senecta tot aerumnis subiectam et undique procellis quassatam cam dolore nos aspicere oporteat atque res in hoc reguo nostro in eum quoque locum sint adductae, ut non solum nostris primatis, rerum etiam S. R. Mtis concionatoribus publice rereque verbum dei a commentis hominum repurgatum profiteri haud quaquam liceat eosque recessus quaerere et in latebras se abdere sit necesse, neque alibi ingenui reritatis professores, si quid tale illis accidat, commodius, quam in ditione Vrae Illmae Domnis perfugia et domicilia consequi queant. hic quoque eximins et eruditas vir Laurentias S. R. Mtis ecclesiastes in eas angustias est redactus in eamque necessitatem adactus, ${ }^{1}$ ) at illi in ditionem

1) Doch hat Laurentius nicht nur seiner erangelischen Gesinuung wegen sein Hofpredigeramt anfgeben müssen. Er, der später ein Schandfleck der polnischen Reformation geworden ist, hat gewisse tible Seiten seines Charakters schon in seiner Stellung am Hofe offenbar werden lasseu. Krakau, den 30 . April 1553 gedenkt B. Pohibel des Osiaudrischen Streites in Rönigsberg und der Ausweisung Mörling und berichtet hier dem Herzoge Albrecht: "Wirt gemelter Morlen 
I. III. Domuis tanquam in certum ecclesiae exulantis hospitium et domicilinm commigrandum esset. Quare eum ego Vrae Illmae Domni diligentissima commendatione commendo rogoque plurimum, ut tandem istine illi tuto per benignitatem V rae Illmae Domnis rersari citra ullum ab bostibus rerbi dei periculam liceat. In maximam adducor spem Vram Illmam Domnem pro ea, qua est erga afflictam ecclesiam roluntate. eum clementia sua esse complexuram et hac in parte opera sua illi non defuturam... Petereoriae 3. Julii 15550.

\section{Herzog Albrecht an Johann Cosmius.}

Venerande sincere nobis dilecte. Accepimus R. Domnis Vrae literas una cum libellis nobis et charissimae coningi nostrae missis, quibus lectis intelleximus, quo ardore $\mathbf{k}$. Domtio V. in propaganda doctrina evangelica laboret, quod ut gratum nobis est et pio homine dignam, ita summae $\mathbf{k}$. Domni V. pro illo munere nobis misso aguntur et habentur gratiae. Daturi operam, at ricissim hanc benerolentiam demereamur, easdem quoque gratias agit illustrissima coniunx nostra, cai munus oblatum fuit gratissimum. Quae porro R. Domtio V. de imposito silentio scribit, ut dolenda sunt, ita in tempus ferenda, quando piorum coetus einsmodi, obnoxins at sit, discriminibus et craci subiceat necesse est. Consolabitur se autem K. Dontio V. eius mercedis, quod omnibus Christi fidelibus commune erit in novissimo die. Quam R. Domnem V. confortare in proposito suo vehementer optamus et percupimus eamque protectioni divinae committimus. Regiomonte 11. Decembris 1550.

\section{Johann Cosmins an Herzog Albrecht.}

Illustrissime princeps. Libellum meum gratum et acceptum faisse Ill. Domui V. magnopere gaudeo. Sed dum nos eum libellum partim cudimus, partim amicis impartimus, ecce

bey vielenn nicht anderst denn vor den Discordiam oder Laurencium, welcher ethwan konr Majt prädicant gewesenn, sich itzo in E. F. G. furstentamb erhelth, geacht vnd angesehenn. Dan gedachter Discordia hatte bei der konn Majt eine ser gate vorhaltunge. Bo war ond ist noch in der schrifft Ber wol erfarenn vnnd gelerdt, jdoch nicht Bo gelerdt, das er seyne zunge hette zugeprauchen wissenn, sonder eynen juern bohes vnd niderenn standes nach seynem gefallenn vnd mudtwillen, keyne maBe gebraucht, geschmehet vnd nit verschonet, alzo das er seynes vnnützes gespeyes ron koniglichem hoffe abgefertiget wardt rnd vielen leuten, welche das wort gottes treulich vnd gern angenommen, durch seynen vnnützen mandt ergernus gegebenn. Eben alzo wirt der Morlein bey vilen vnd nit geryngen leuten der form rnd gestalt abgemalet, wissen auch viel, das es E. F. D. lange genugk mit jm vorsucht vand viel geduldet'. 
ibi prodierunt aliae literae ex cancellaria S. R. Itis, in quibus miro quodam ac stapendo vinculo archiepiscopus cum suis episcopis obstrinxit Sacram Mtem Suam ad tutandam ecclesiam Romanam. Quid enim non obstringerent. cum Maiestatem suam boc tempore coronationis ita in potestate habebant, ut etiam ad percussionem digitorum quoscunque rellent orthii modos saltaret, nihil cum non faceremus, modo coronationem consequeremar. Ac cum haec ex solo archiepiscopo potissimum pendebat, nihil tam magnum ac arduum peteret, cuius repulsam reportaret. Cumque illi a Sacra Mte dabatur episcopatas Cracoviensis. ${ }^{1}$ ) quod se scilicet tam facilem ad coronandum praebuisset, renuit episcopatum. id saltem oravit, nt haic Mtas Regia daret, cui ille vellet, datusque est episcopatus ad arbitrium archiepiscopi homini vitae contaminatissimae moribus spurcissimis a sacris literis alienissimo ${ }^{2}$ ) qui apertis sermonibus de Christo domino eadem, o horrendum, dicere solebat, quae de Mahometo. Renuens autem archiepiscopus episcopatum Cracoriensem id sibi pro episcopatu dari a maiestate sua oravit, ut maiestas scilicet sua literis propria mana subscriptis sub eodem, quo regno iuravit, iuramento sacrosancto sese obligaret, se Romanam ecclesiam cum omnibus sujs assertionibus, consuetudinibus, traditionibus ete. defensuram, in eosque omnes, qui Romano pontifici contrarie vellent, carceribus, exilio etc. animadversuram. Quarnm literarum Illnae Domni copiam summo animi mei dolore mitto. ${ }^{3}$ ) Sed quia deus omnipotens pro sua admirabili sapientia eos plerumque caecutire facit, qui se per mundi huius prudentiam videre putant, in hoc archiepiscopum cum episcopis caecavit, qui scilicet quotiescunque Romanae ecclesiae mentionem in his literis faciunt, toties et ecclesiae catholicae et apostolicae meminerunt, ut non tam piis et erangelicis viris hae literae maiestatis regiae noceant, qui semper catholicam ecclesiam apostolicam appellant eiusque doctrinam ac disciplinam nempe cum evangelio consentientem anhelant, quam illis ipsis episcopis, qui aperto marte in omuibus suis et actionibus et doctrinis ecclesiam catholicam ae apostolicam oppugnant, sint nocitarae, si quis eas paulo diligentius ad amussim canonis et verbi dei dimetiri ac excutere studeret. Eo enim impietatis ac impadentiae per omnium rerum dissolutam licentiam perveverunt, ut ne umbram quidem catholicae ecclesiae canonum, quos aperta vi violant, retinere videantur. Si igitur maiestas sua obstrinxit storben.

1) Bischof Samuel Jaciejowski war am 26. Oktober 1550 ver-

$\left.{ }^{2}\right)$ Andreas Zebrzydowski. Ueber sein Leben vgl. Arta hist. res gestas Poloniae illastrantia I, 481.

3) Das königliche Mandat rom 12. Dezember 1550. 
se in eos animadrersuram, qui catholicae et apostolicae ecclesiae non paruerint, certe imprimis suae saereritatis fasces ac secures in eos ipsos episcopos ac canonicos etc. depromat oportet, qui manifestam vim catholicae ecclesiae plas trecentis articulis, ut iam a me observatum et notatam est, inferunt.

lamque me haic negotio accinxi, at id archiepiscopo cum omnibus episcopis liquido et aperte demonstrem, imprimis Romanam ecclesiam per omnia contrariam esse ecclesiae catholicae et apostolicae, ${ }^{1}$ ) porro plus huic ecelesiae debemus, quam sanctam catholicam et apostolicam in symbolo confitemur, quam Romanae, cuins nulla est apud sanctos doctores publica professio, imo iam multi vocant Babylonicam ut S. Petrus et Hieronymus et alii fere omnes, deinde at demonstrem episcopos omnes obnoxios esse illis ipsis literis regiis ac exilio, cum apertissimi hostes canonum apostolorum ac ecclesiae catholicae sint. Et quamquam haec omnia in ea oratione nostra, quam nuper ad Julium III pontificem Romanum seripsi, pertracto, at si saperent, contenti esse possent, ne maior impietas eorum in lucem clariorem prodeat, tamen cum ad cor redire hac nostra oratione nolunt, experientar deinceps quiddam magis et serium et aculeatum, at alioquin tandem resipiscant.

Sed quia hic Cracoviae cautum severe est sub decem talentis auri pari, ne quid imprimatar, nisi prius doctorum in collegio censaram subeat, qui tam acate in verbo dei vident, quam ille vetustas Satarnus lippientibus oculis etc., vixque furtim obtinui, nt rari non longe a Cracovia haec oratio ad Julium III imprimeretar idque incredibili meo sumptu ac sadore, ut facile Illma Domtio Vra colligere poterit etc. Proinde nil magis necessitati, honestati ac laudi Illmae Domnis Vrae consonum esset quam Regiomontem abandare, quemadmodum optimis viris et doctrinis abundat, et characteribus graecis ac latinis haebraicisque, ut illic excudi possent in promptn ea, quae in gloriam Christi ac ecclesiae aedificationem, diaboli etiam vastitatem esse possent. Nam in Germaniam haec mittere est partim longissimi itineris partim insolentis et rari, haee autem maturitatem imprimendi efflagitant, at tempestive homines impietatem episcoporum ac totius Romanae ecclesiae agnoscant, priusquam haec eorum tyrannis radices in profundum agant. Quapropter Illmam Domnem V. at ehristianissimam principem oro, faciat me prima occasione certiorem, an illic Regiomonte imprimi debeat id, in quo nunc novo stadio excubabo brevique parturiam, at omnibus constet literas hasce regias, quarum Illmae Dom ${ }^{\text {ni }}$

1) Vgl. Wotschke, Laskis Reinigangseid. Korrespon lenzblatt des Vereins für die Geschichte der ev. Kirche Schlesiens X S. 120 
Vrae exemplar mitto. non nobis. contra quos illas impetraverant, sed illis ipsis internecionem ac exilium minari, ut illis id plane accidat, quod Maxentio a Constantino victo, qui dum pontem ad decipiendum et submergendum bostem deiecit et artificione disposuit, ipsemet postea rictus in easdem decipulas cum exercitu incidit inciditque in foream, quam fecit. Tandem me gratiae Illmae Domni lrae commendo illamque Christo domino eiusque sacrificio ac regno, quod sustinet in dextera patris sui uostrique, deroveo, ut ille soo olocausto perpetuo omnia studia ac conatus pios Illmae Domnis Vrae in landem saam saaeque ecclesiae promoveat ac ampliet. Dat. Cracoviae die Joannis (12. März) 15う́1. Eiusdem Illmae Domnis Vrae Joannes Cosmins concionator S. M. ${ }^{1}$ )

1) Die herzogliche Antwort habe ich nicht anffinden können. 